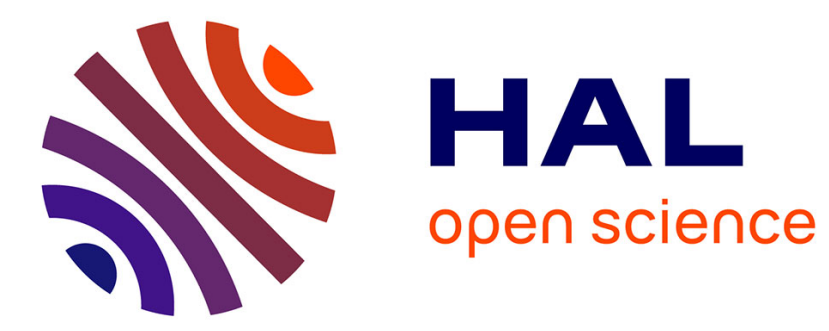

\title{
Interaction avec un agent motivationnel pour soutenir le changement de comportement
}

\author{
Antoine Louvet, Brian Ravenet, Céline Clavel, Nicolas Sabouret
}

\section{To cite this version:}

Antoine Louvet, Brian Ravenet, Céline Clavel, Nicolas Sabouret. Interaction avec un agent motivationnel pour soutenir le changement de comportement. Conférence Internationale Francophone sur l'Interaction Homme-Machine (IHM 20.21), Apr 2021, Metz, France. 10.1145/3451148.3458647 . hal-03505236

\section{HAL Id: hal-03505236 \\ https://hal.science/hal-03505236}

Submitted on 30 Dec 2021

HAL is a multi-disciplinary open access archive for the deposit and dissemination of scientific research documents, whether they are published or not. The documents may come from teaching and research institutions in France or abroad, or from public or private research centers.
L'archive ouverte pluridisciplinaire HAL, est destinée au dépôt et à la diffusion de documents scientifiques de niveau recherche, publiés ou non, émanant des établissements d'enseignement et de recherche français ou étrangers, des laboratoires publics ou privés. 


\title{
Interaction avec un agent motivationnel pour soutenir le changement de comportement
}

\author{
Interaction With a Motivational Agent to Support Behaviour Change
}

\author{
Antoine Louvet \\ louvet@limsi.fr \\ LISN, Université Paris-Saclay, CNRS \\ Orsay, France \\ Brian Ravenet \\ Brian.Ravenet@limsi.fr \\ LISN, Université Paris-Saclay, CNRS \\ Orsay, France
}

\author{
Céline Clavel \\ celine.clavel@limsi.fr \\ LISN, Université Paris-Saclay, CNRS \\ Orsay, France \\ Nicolas Sabouret \\ nicolas.sabouret@limsi.fr \\ LISN, Université Paris-Saclay, CNRS \\ Orsay, France
}

\begin{abstract}
We are developing a virtual agent based on the Motivational Interviewing method to help elderly and inactive individuals change their behavior. The agent engages users in a conversation that aims to highlight reasons why they might want to increase their level of physical activity. In this work, we describe our approach to computerized adaptation of Motivational Interviewing. A study evaluates the outcome of this approach and the system as a whole.
\end{abstract}

\section{CCS CONCEPTS}

- Human-centered computing $\rightarrow$ Interaction design; User studies.

\section{KEYWORDS}

Conversational agent, Interaction method, Motivational Interviewing, Typology, Evaluation Study

\section{RÉSUMÉ}

Nous développons un agent virtuel qui s'inspire de la méthode de l'Entretien Motivationnel pour soutenir des individus âgés et inactifs dans un changement de comportement positif. L'agent engage les utilisateurs dans une conversation qui a pour objectif de mettre en évidence les raisons qui pourraient les inciter à augmenter leur niveau d'activité physique. Dans ce travail, nous décrivons notre approche pour effectuer une adaptation informatisée de l'Entretien Motivationnel. Une étude évalue le résultat de cette démarche et le système dans son ensemble.

\section{MOTS-CLÉS}

Agent conversationnel, Méthode d'interaction, Entretien Motivationnel, Typologie, Etude d'évaluation

Permission to make digital or hard copies of part or all of this work for personal or classroom use is granted without fee provided that copies are not made or distributed for profit or commercial advantage and that copies bear this notice and the full citation on the first page. Copyrights for third-party components of this work must be honored

For all other uses, contact the owner/author(s).

IHM '21 Adjunct, April 13-16, 2021, Virtual Event, France

(c) 2021 Copyright held by the owner/author(s).

ACM ISBN 978-1-4503-8377-6/21/04.

https://doi.org/10.1145/3451148.3458647

\section{ACM Reference Format:}

Antoine Louvet, Céline Clavel, Brian Ravenet, and Nicolas Sabouret. 2021. Interaction avec un agent motivationnel pour soutenir le changement de comportement. In 32 e Conférence Francophone sur l'Interaction HommeMachine (IHM '21 Adjunct), April 13-16, 2021, Virtual Event, France. ACM, New York, NY, USA, 6 pages. https://doi.org/10.1145/3451148.3458647

\section{INTRODUCTION}

L'allongement de l'espérance de vie a pour conséquence l'augmentation des maladies chroniques qui constituent aujourd'hui la première cause de mortalité dans le monde [22]. Ces importants enjeux sanitaires ont motivé le développement de mesures de prévention comme la promotion de la pratique d'une activité physique régulière limitant le développement de certaines pathologies chroniques [10].

Parmi les méthodes utilisées afin d'inciter les individus à faire de l'activité physique, la méthode de l'Entretien Motivationnel (EM) [20] a fait ses preuves en tant que moyen efficace pour induire un changement de comportement [18]. Elle repose sur la construction d'une relation de collaboration dans laquelle le thérapeute cherche à comprendre les envies et les difficultés de l'individu afin de construire avec lui un plan d'action personnalisé. Une part essentielle de l'EM consiste à explorer la subjectivité de l'individu à travers des questions ouvertes qui permettent d'identifier ses objectifs et les valeurs personnelles (e.g. la conformité, l'indépendance, la prudence, etc.) sur lesquelles ces objectifs reposent [20]. Cette exploration permet à l'individu de développer une ambivalence, c'est-à-dire la prise de conscience d'une incohérence entre un de ses objectifs (e.g. rester en bonne santé), ou une de ses valeurs personnelles, et le comportement qu'il adopte (e.g. ne pas faire suffisamment d'activité physique). Au fur et à mesure du renforcement de l'ambivalence, il devient de plus en plus nécessaire à l'individu de la résoudre, notamment par l'alignement de son comportement avec ses objectifs (e.g. faire davantage d'activité physique pour rester en bonne santé). Pour soutenir le développement d'une ambivalence, le thérapeute reformule les idées exprimées par le patient afin de susciter chez lui une réflexion sur le lien existant entre ses objectifs et ses valeurs personnelles d'une part, et son comportement d'autre part [20]. Une reformulation est dite simple lorsque son sens est très proche de l'idée initiale, il peut alors s'agir de répéter ce que vient 
de dire le patient. À l'inverse, une reformulation est dite complexe lorsqu'elle se distingue de l'acte de langage employé par le patient afin de lui apporter une nouvelle perspective sur ce qu'il a exprimé. Les Reformulations Complexes nécessitent néanmoins une bonne compréhension des objectifs et des valeurs de l'individu.

Récemment, des recherches se sont intéressées à développer des systèmes capables de conduire un Entretien Motivationnel de façon automatisée [8, 14, 16, 24, 27, 31]. Les interventions de santé informatisées ont montré une certaine efficacité afin d'inciter les individus à faire davantage d'activité physique [9]. Ce type d'intervention peut se montrer utile afin de promouvoir divers comportements de santé auprès d'un large public, particulièrement lorsque le personnel de santé n'est pas disponible. Cependant, les adaptations informatisées de l'EM n'utilisent généralement qu'une minorité des composants de cette méthode et cela limite la capacité de ces systèmes à induire un changement de comportement [32]. Particulièrement, les systèmes cités effectuent une exploration superficielle de la subjectivité de l'utilisateur ce qui peut expliquer leur utilisation de Reformulations Simples alors que les Reformulations Complexes sont privilégiées afin de soutenir le développement d'une ambivalence chez les individus [21].

Dans le cadre de notre projet de recherche, nous nous intéressons à concevoir un agent virtuel qui promeut la pratique d'une activité physique régulière auprès d'individus peu actifs et âgés en utilisant la méthode de l'Entretien Motivationnel. Cette population n'a pas encore été considérée dans les adaptations informatisées de l'EM. Dans ce travail, nous présentons notre système et l'approche que nous avons adoptée afin de concevoir l'ensemble des choix prédéfinis proposés aux utilisateurs pour interagir avec le système. Ces actes de dialogue ont pour but de permettre l'exploration du point de vue de l'utilisateur afin que le système soit en capacité d'effectuer des Reformulations Complexes. Dans une étude, nous évaluons le résultat de notre approche et la perception des utilisateurs envers le système.

\section{TRAVAUX SIMILAIRES}

L'Entretien Motivationnel s'appuie sur une discussion au cours de laquelle l'individu exprime son point de vue. En conséquence, certaines adaptations informatisées de l'EM se sont attachées à garantir la libre expression de l'utilisateur en lui permettant de s'exprimer en langage naturel $[8,14,16]$. Cependant, le traitement du langage naturel cause encore de nombreux échecs de compréhension, même lorsqu'il s'agit d'interactions simples [12]. Dans le cadre d'un entretien reposant sur une interaction complexe d'une durée relativement longue, la qualité de l'intervention peut s'en trouver sévèrement affectée [14]. Dans cette étude, les échecs de compréhension et la mauvaise gestion des tours de parole ont été si récurrents que les participants ont préféré assister à un monologue présentant les bénéfices de l'activité physique plutôt que d'interagir avec un robot s'inspirant de l'EM. Afin de contourner les limites du traitement du langage naturel, une approche consiste à laisser l'utilisateur s'exprimer librement sans que cela ne soit pris en compte par le système [8]. Dans ce cas, les réactions du système suivent un script déterminé dont le déroulement n'est nullement influencé par les entrées de l'utilisateur. Néanmoins, cette approche semble incompatible avec l'esprit de l'EM qui appelle à la personnalisation du discours en fonction du point de vue de l'individu [20]. Lorsque le système prend effectivement en compte les entrées en langage naturel, les questions employées impliquent nécessairement des réponses courtes et restreintes à un faible nombre de possibilités [16]. Par conséquent, l'exploration du point de vue de l'utilisateur est limitée car il n'a pas la possibilité d'exprimer ses objectifs et les valeurs qui les supportent. Depuis récemment, des efforts conséquents sont mis en œuvre afin de permettre la conception d'EM informatisés autorisant des entrées en langage naturel tout en satisfaisant au principe de libre expression [25] bien que la plupart de ces systèmes ne dépassent pas la phase pilote [28]. En attendant l'aboutissement de ces travaux, dont les résultats pourraient ouvrir une nouvelle ère dans le développement des interventions de santé informatisées, de nombreux travaux adoptent un compromis en proposant à l'utilisateur un ensemble de choix de réponses prédéfinis [24, 27, 31]. L'utilisateur ne peut donc pas s'exprimer librement, mais le dialogue est adapté aux réponses de chaque individu.

Les résultats de ces recherches suggèrent que la restriction des entrées de l'utilisateur n'induit pas une perception négative du système. Cette méthode d'interaction permet de garantir la compréhension de l'agent tout au long de l'entretien ce qui est particulièrement important pour une population peu à l'aise avec la technologie comme les personnes âgées de plus de 55 ans [1]. De plus, cette méthode permet d'éviter toute déviation de la structure de l'Entretien Motivationnel et elle peut soutenir l'utilisateur dans le développement d'une ambivalence en l'incitant à la réflexion face aux options de réponse qui lui sont proposées [31]. Cependant, les systèmes cités utilisent uniquement des Reformulations Simples (i.e. répétition de ce qu'a dit l'utilisateur) alors que les Reformulations Complexes sont recommandées pour soutenir le développement d'une ambivalence [21]. Bien que l'utilisation d'actes de dialogue prédéfinis constitue en soit un écart par rapport au principe de libre expression de l'Entretien Motivationnel, il semble possible de se rapprocher davantage de cette méthode en s'efforçant de mettre en œuvre l'ensemble de ses techniques.

\section{VUE D'ENSEMBLE DU SYSTÈME}

Notre système est construit autour d'un arbre de dialogue avec des choix de réponses prédéfinis. La discussion est à l'initiative de l'agent et l'utilisateur choisit sa réponse à travers l'interface graphique (Fig. 1). La suite de l'entretien dépend de la réponses sélectionnée.

\subsection{Agent virtuel}

L'engagement dans l'Entretien Motivationnel implique la formation de bonnes relations entre le thérapeute et l'individu [20]. Pour favoriser l'émergence de cette relation entre un utilisateur et un système, le système peut s'incarner à travers un agent anthropomorphique [3]. L'agent est donc représenté par un modèle graphique qui se veut réaliste. En effet, les agents réalistes induisent une évaluation plus positive que les agents peu réalistes [33] et ils sont perçus comme davantage sociaux [23]. L'agent prend l'apparence d'une personne d'environ 60 ans afin de présenter aux utilisateurs un interlocuteur qui leur ressemble et qui est donc plus à même de les influencer [4]. De plus, il illustre les effets bénéfiques de l'activité physique en étant mince et en bonne forme physique afin d'être 


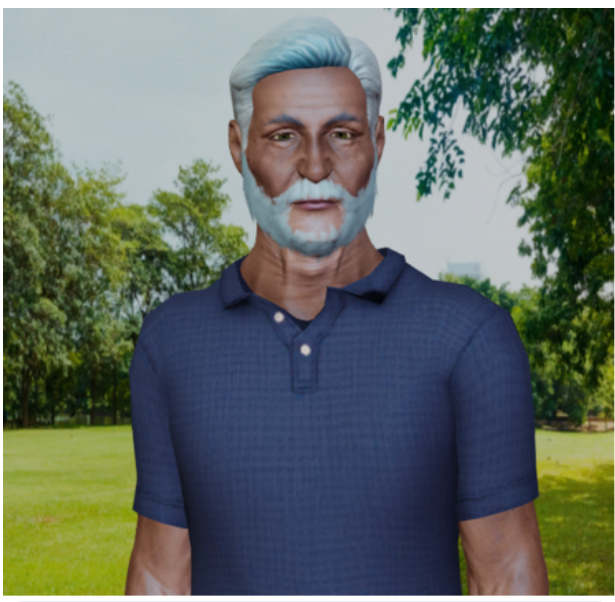

J'aimerais me motiver à faire plus d'activité physique.

J'aimerais trouver des moyens de faire plus d'activité physique.

J'aimerais faire le point sur mon activité physique.

Figure 1: L'agent, Jean, a demandé à l'utilisateur ce qu'il espère de la discussion.

perçu comme un modèle à suivre [4]. Sa voix a été enregistrée par un acteur professionnel, les voix humaines étant soit préférées aux voix synthétiques [6], soit jugées de manière similaire à une voix synthétique de très bonne qualité [7]. Le comportement non-verbal de l'agent se déclenche en fonction de mots clés contenus dans ses actes de dialogue. À chaque mot clé sont associées une expression faciale et une gestuelle cohérente avec son discours. Cette caractéristique favorise la confiance de l'utilisateur envers le système [17], sa satisfaction envers l'interaction [29] et son sentiment d'être en présence d'un partenaire social crédible [15]. Lorsque l'agent exprime verbalement son approbation, il peut hocher la tête, sourire, lever les sourcils, se pencher légèrement sur le côté ou plus généralement, utiliser une combinaison de ces comportements non-verbaux [5]. L'agent exprime aussi de la compassion, de l'étonnement et de la curiosité mais il évite d'exprimer toute émotion négative qui pourrait nuire à la relation avec l'utilisateur [20].

Notre agent utilise les quatre techniques de l'Entretien Motivationnel visant à soutenir le développement d'une ambivalence [20]. Il pose des questions ouvertes ayant pour objectif d'explorer les objectifs et les valeurs personnelles de l'utilisateur (e.g. "Qu'est-ce qui pourrait arriver de mieux si vous faisiez plus d'activité physique ?"). Il utilise des Reformulations Complexes qui vont au-delà de la répétition de l'idée exprimée par l'utilisateur. Il résume les options de réponse sélectionnées afin de mettre en évidence les raisons de faire de l'activité physique que l'utilisateur a lui-même évoqué et il emploie des affirmations pour renforcer la confiance de l'utilisateur en ses capacités à changer (e.g. "Vous êtes déterminé à atteindre vos objectifs!").
Agent: Je suis curieux de connaitre votre avis : selon vous, quel est l'intérêt de faire de l'activité physique? [Question ouverte]

Utilisateur: Protéger sa santé, réduire les risques de maladie et retarder le vieillissement du corps (Choix d'une catégorie de raison)

Utilisateur: Réduire les risques de développer une maladie chronique (Choix d'une raison)

Agent: Vous ne voulez pas que quelque chose de terrible vous arrive. Qu'est-ce que vous redoutez le plus? [Reformulation/Question ouverte]

Utilisateur: Ce serait que la maladie m'accapare, que mon quotidien se construise autour d'elle. (Choix d'une catégorie de valeur)

Utilisateur: Le pire serait de ne plus avoir confiance en mon futur, de devenir fataliste. (Choix d'une valeur)

Agent: Vous ne voulez pas vous résigner et penser qu'il n'y a plus rien à faire. [Reformulation]

FiguRE 2: Retranscription partielle d'une interaction. Les techniques de l'EM utilisées sont indiquées entre crochets.

\subsection{Actes de dialogue de l'utilisateur}

Une difficulté du développement d'un agent virtuel dont les entrées sont restreintes réside dans la conception d'options de réponse représentatives des thèmes que les utilisateurs voudront aborder. Cela est d'autant plus vrai lorsque les questions de l'agent virtuel se préoccupent des motivations profondes des individus. Pour les déterminer, nous nous sommes appuyés sur la lecture d'entretiens et de témoignages afin d'identifier les raisons pour lesquelles les individus disent percevoir un intérêt à la pratique d'une activité physique. Dans un premier temps, nous avons analysé 21 EM retranscrits traitant de l'activité physique ou de sujets en lien (e.g. contrôle du poids, gestion du diabète, faible estime de soi) et menés par des praticiens formés à l'Entretien Motivationnel [2]. Une trentaine d'objectifs ont été identifiés de cette manière. Dans un second temps et afin d'identifier des objectifs plus spécifiques à la population que nous ciblons, nous avons recherché des témoignages déjà existants de personnes actives de plus de 55 ans sur des sites web ou des groupes en lien avec l'activité physique. Une dizaine d'objectifs supplémentaires ont émergé. Certains de ces objectifs n'étaient pas soutenus par des preuves scientifiques convaincantes (e.g. "augmenter la libido"), ils ont donc été exclus de la liste finale afin de ne pas induire des attentes irréalistes chez les utilisateurs du système. Les 37 objectifs de la liste finale ont ensuite été regroupés en 15 objectifs plus larges pour faciliter la navigation dans l'interface (e.g. "réduire les risques de Parkinson/d'Alzheimer/de cancer du côlon/de cancer du sein" dans un objectif "réduire les risques de maladie grave"). Pour cette même raison, ces objectifs ont été divisés en cinq grandes catégories : la protection de la santé, le développement du corps, le bien-être psychologique, l'opportunité de socialiser et l'amélioration de la qualité de vie. Lors de l'interaction avec l'agent virtuel, l'utilisateur doit d'abord sélectionner une de ces catégories afin d'accéder aux objectifs correspondants. Il peut aussi revenir en arrière pour changer sa sélection.

L'Entretien Motivationnel s'intéresse aussi à explorer les valeurs qui expliquent l'importance d'un objectif aux yeux de l'individu qui l'a évoqué. Puisque l'analyse des entretiens retranscrits ne pouvait pas faire émerger l'ensemble des possibilités, nous avons préféré nous appuyer sur une compilation de 83 valeurs personnelles [19]. 
Dans le cadre d'un EM, le thérapeute peut présenter cette compilation à un individu qui éprouve des difficultés à définir les valeurs qui sont importantes à ses yeux [20]. Pour chacun des 15 objectifs, nous avons procédé par élimination en excluant les valeurs qui étaient manifestement peu cohérentes avec un objectif donné (e.g. l'objectif "Réduire les risques de développer une maladie chronique" et la valeur "Coopération"). Pour chaque objectif, 4 à 8 valeurs ont ainsi été jugées pertinentes par deux chercheurs en psychologie. Sur la base de ces valeurs, les options de réponse ont ensuite été conçues en prenant en exemple les formulations utilisées par les patients dans les retranscriptions d'entretien. Les options de réponse ont finalement été regroupées en 2 à 3 catégories thématiques par objectif afin de limiter le nombre de choix affiché à la fois sur l'interface. L'utilisateur doit d'abord sélectionner une catégorie pour avoir accès aux options de réponses qui déclencheront une réaction de l'agent. Il a aussi la possibilité de changer sa sélection.

Cette approche a permis d'automatiser une phase essentielle de l'EM consistant à mieux comprendre le point de vue de l'individu. Dans notre système, cette exploration du point de vue de l'individu permet l'utilisation de Reformulations Complexes (Fig. 2). Notre méthodologie de conception des options de réponse implique une part importante de subjectivité et par conséquent, nous avons évalué son résultat directement auprès des utilisateurs. Cette étude a aussi été l'occasion de chercher des pistes d'amélioration du système dans son ensemble.

\section{4 ÉTUDE D'ÉVALUATION}

\subsection{Mesures}

Le niveau d'activité physique des participants a été mesuré à l'aide du questionnaire de Ricci et Gagnon utilisé par l'Assurance Maladie [30]. Ce questionnaire catégorise les individus selon trois niveaux d'activité physique : inactif, actif et très actif.

Un objectif de ce travail est de proposer à l'utilisateur un ensemble d'options de réponse qui lui permet d'exprimer fidèlement les idées qu'il aurait partagé s'il avait pu directement parler à l'agent virtuel. A cette fin, un item s'intéressait à la fidélité des options de réponse sélectionnées au regard des idées que l'utilisateur voulait exprimer (i.e. Les options de réponse que j'ai sélectionnées étaient en accord avec ce que je voulais dire), un item mesurait l'exhaustivité des options de réponse (i.e. Les options de réponse que j'ai sélectionnées étaient des choix par défaut) et un item mesurait la pertinence de leur formulation (i.e. Les options de réponse étaient formulées de manière naturelle, comme j'aurais pu les dire moi-même.). Le score de l'item "Les options de réponse que j'ai sélectionnées étaient des choix par défaut" a été inversé lors des analyses. Pour explorer dans quelle mesure la sélection des options de réponse était aisée, un item s'intéressait à la clarté des formulations (i.e. Les options de réponse étaient formulées de façon claire et compréhensible) et un autre item mesurait leur bonne catégorisation parmi les différentes catégories de réponse proposées (i.e. Les options de réponse que j'ai sélectionnées étaient faciles à trouver parmi les autres options de réponse). Les participants répondaient à l'aide d'une échelle de Likert en 5 points, de « Tout à fait en désaccord » à « Tout à fait d'accord ». Afin de compléter et de corriger les actes de dialogue prédéfinis qui permettaient aux participants de s'exprimer, ces derniers avaient la possibilité d'écrire librement
TABLE 1: Mesures concernant les options de réponse proposées à l'utilisateur.

\begin{tabular}{ccl}
\hline Item (Les options de réponse étaient...) & Moyenne & Écart-type \\
\hline ...en accord avec ce que je voulais dire. & 4.17 & 0.76 \\
(R) ...des choix par défaut. & 3.58 & 0.91 \\
...formulées de façon claire. & 4.41 & 0.87 \\
...formulées de manière naturelle. & 3.59 & 1.09 \\
...faciles à trouver. & 4.10 & 0.94 \\
\hline
\end{tabular}

les idées qu'ils auraient voulu évoquer mais qui étaient mal ou non représentées dans l'ensemble de choix à leur disposition. Cette option de réponse était disponible à tout instant. Son utilisation n'influençait pas les réactions du système car la sélection d'une réponse prédéfinie restait nécessaire pour déclencher une réponse de l'agent.

Un questionnaire a évalué la perception des participants envers le système et l'agent [13]. A l'aide d'une échelle de Likert en 5 points, de « Tout à fait en désaccord » à « Tout à fait d'accord », les participants indiquaient leur degré d'accord avec des affirmations regroupées dans les 9 dimensions suivantes : 1. Appréciation de l'interaction (APP), 2. Présence sociale perçue (PRE), 3. Sociabilité perçue chez l'agent (SOC), 4. Confiance envers l'agent (CON), 5. Anxiété ressentie lors de l'interaction (ANX), 6. Attitude envers le système (ATT), 7. Intention d'utiliser le système (INT), 8. Facilité d'utilisation du système (FAC), 9. Utilité perçue du système (UTI). Le score de la dimension "Anxiété ressentie lors de l'interaction" a été inversé lors des analyses. De plus, les participants pouvaient rédiger un commentaire de façon libre afin d'indiquer les éléments du système qui leur ont déplu.

\subsection{Procédure}

L'étude a été auto-administrée. Les participants ont été recrutés sur les réseaux sociaux, sur des groupes destinés aux personnes de plus de 50 ans. Les participants n'étaient pas rémunérés et la passation de l'expérimentation s'est déroulée à travers un questionnaire en ligne, via leur ordinateur personnel.

Après avoir cliqué sur le lien de l'étude, les participants répondaient au questionnaire d'activité physique. Ils étaient ensuite redirigés vers un site Internet hébergeant le système où une courte vidéo leur permettait de comprendre comment interagir avec l'agent virtuel. A la fin de l'interaction, les participants étaient invités à revenir au questionnaire. Leur perception envers les options de réponse était recueillie puis leur perception envers l'agent et le système était mesurée. Enfin, ils indiquaient leur âge et leur genre puis nous leur présentions les tenants et aboutissants de notre travail avant de les remercier pour leur participation.

\subsection{Résultats}

Il y a eu 29 participants dont 15 femmes et 14 hommes. La majorité des participants avaient plus de 55 ans (19), tandis que les 45-54 ans (5), les 35-44 ans (2) et les moins de 34 ans (3) étaient minoritaires. La plupart des participants étaient actifs (15) ou inactifs (13) et seulement 1 participant était très actif. 
Concernant la perception des options de réponse, tous les items ont une moyenne supérieure à 3.5 sur une échelle en 5 points (Table 1). Il n'existe pas de différence significative entre les personnes de moins de 55 ans et les personnes de plus de 55 ans. L'option "Réponse libre" a seulement été utilisée une fois.

Concernant la perception du système et de l'agent, la plupart des dimensions ont une moyenne supérieure à 3 sur une échelle en 5 points (Table 2). Seule la dimension "Présence sociale" obtient un faible score de $2.64(\mathrm{EC}=1.15)$ qui est cohérent avec les commentaires des participants sur le réalisme comportemental de l'agent. En effet, plusieurs d'entre eux ont fait état d'animations saccadées ou peu réalistes et de l'enchaînement peu naturel de certains enregistrements audio relatifs à la voix de l'agent.

Des tests de Mann-Whitney ont été utilisés afin de comparer l'évaluation du système entre les participants actifs (15) et ceux inactifs (13). L'unique participant très actif a été exclu de ces analyses. Les participants inactifs ont une attitude plus positive envers le système $(\mathrm{M}=4, \mathrm{EC}=0.5)$ que les participants actifs $(\mathrm{M}=3.36, \mathrm{EC}=$ $0.81), U=37.5, p=0.05$. De manière tendancielle, les participants inactifs trouvent le système davantage utile $(\mathrm{M}=3.71, \mathrm{EC}=0.64)$ que les participants actifs $(\mathrm{M}=2.98, \mathrm{EC}=0.94), \mathrm{U}=38, \mathrm{p}=0.06$. Dans les commentaires libres, certains participants ont indiqué qu'ils ne voyaient pas d'intérêt à utiliser le système. Tous ces participants (5) étaient actifs.

Le genre des participants n'a pas influencé leur perception des options de réponse ou du système. En revanche, les participants âgés de plus de 55 ans $(\mathrm{M}=3.53, \mathrm{EC}=0.57)$ ont eu moins de facilité à utiliser le système que les participants âgés de moins de 55 ans $(\mathrm{M}=4.06, \mathrm{EC}=0.49), \mathrm{U}=43, \mathrm{p}=0.02$.

\subsection{Discussion}

Les scores élevés des items relatifs aux options de réponse ainsi que le très faible taux d'utilisation de l'option de réponse libre lors de l'interaction suggèrent que l'ensemble de choix prédéfinis est adapté à notre population. Cependant, on ne peut écarter la possibilité que les participants aient préféré sélectionner un choix de réponse qui se rapproche de l'idée qu'ils voulaient exprimer plutôt que de faire un effort rédactionnel afin d'indiquer précisément le fond de leur pensée. En effet, les individus ont tendance à privilégier les solutions les moins coûteuses cognitivement [11]. Par ailleurs, le faible échantillon de cette étude appelle à la confirmation de ces résultats lors des études suivantes.

L'âge des participants n'a pas influencé leur évaluation des options de réponse. Cela peut s'expliquer par la grande diversité des thématiques abordées dans les choix prédéfinis. Alors que certains objectifs proposés sont spécifiques à une population plus âgée (e.g. Réduire les risques liés au vieillissement), la plupart d'entre eux peuvent servir de base motivationnelle pour une population plus large (e.g. Faire partie d'un groupe pour faire des rencontres).

Le questionnaire s'intéressant à l'évaluation du système et de l'agent met en évidence la perception globalement positive des participants envers le dispositif. Il a tout de même permis d'identifier des pistes d'amélioration. Comme en témoignent les scores à la dimension "Présence sociale perçue", le comportement non-verbal de l'agent n'a pas convaincu les participants. Cela peut être dû à l'absence d'animation de repos de l'agent à cause d'un problème
TABLE 2: Mesures concernant la perception de l'agent virtuel et du système

\begin{tabular}{ccl}
\hline Dimension & Moyenne & Écart-type \\
\hline APP & 3.65 & 1.00 \\
PRE & 2.64 & 1.15 \\
SOC & 3.76 & 0.85 \\
CON & 3.25 & 1.10 \\
(R) ANX & 4.14 & 1.11 \\
ATT & 3.49 & 0.88 \\
INT & 3.03 & 1.12 \\
FAC & 3.71 & 1.26 \\
UTI & 3.18 & 0.96 \\
\hline
\end{tabular}

technique mais aussi à une mauvaise synchronisation entre la gestuelle de l'agent et son discours. De manière très claire, certains enregistrements de la voix de l'agent méritent d'être retravaillés car ils ont brisé l'immersion dans l'interaction. Des efforts pourraient aussi être faits afin de garantir une bonne expérience utilisateur chez les participants plus âgés qui rapportent des scores plus faibles à la dimension "Facilité d'utilisation du système" par rapport aux autres participants. Les points considérés concernent la vidéo tutorielle présentée avant l'interaction ainsi que la disposition et la taille des boutons servant à la sélection d'une option de réponse. Il n'existe pas de différence entre les hommes et les femmes dans l'évaluation du système et de l'agent. Si le développement d'un agent féminin a été envisagé, ce résultat suggère qu'il n'est pas nécessaire de présenter aux femmes un agent virtuel qui leur est similaire pour favoriser leur perception positive du système.

\section{CONCLUSION}

L'étude présentée dans cet article a permis de valider le caractère adapté, pour une population âgée et inactive, des options de réponse et du système d'EM dans son ensemble. La suite de nos travaux consistera à apporter les modifications évoquées afin d'améliorer la qualité de l'interaction. L'interaction sera ensuite prolongée pour intégrer l'ensemble de la structure et des techniques de l'Entretien Motivationnel. À cette fin, l'agent doit être capable d'identifier les obstacles au changement de comportement perçus par les utilisateurs (e.g. manque de temps) puis d'améliorer la confiance qu'ils ont en leur capacité à les surmonter. Il doit aussi pouvoir discuter des actions envisagées par les utilisateurs dans le but de concrétiser le changement positif de comportement. Les recherches similaires évaluent rarement la capacité du système à induire un changement de comportement sur le long terme [26]. Afin d'explorer l'efficacité réelle de notre système, il fera l'objet d'une étude longitudinale mesurant l'évolution du niveau d'activité physique des utilisateurs.

\section{REMERCIEMENTS}

Cette recherche est soutenue par le projet ANR CoPains ("Cognitive Planning in Persuasive Multimodal Communication"). Nous remercions l'entreprise DAVI pour son travail sur la partie technique du système. 


\section{RÉFÉRENCES}

[1] Awais Ahmad and Peter Mozelius. 2019. Critical factors for human computer interaction of ehealth for older adults. In Proceedings of the 2019 the 5th International Conference on e-Society, e-Learning and e-Technologies. 58-62.

[2] AlexanderStreet. 2021. Counseling and Psychotherapy Transcripts : Volume II Retrieved from https ://search.alexanderstreet.com (2021).

[3] Amy L Baylor. 2009. Promoting motivation with virtual agents and avatars : role of visual presence and appearance. Philosophical Transactions of the Royal Society B : Biological Sciences 364, 1535 (2009), 3559-3565.

[4] Amy L Baylor. 2011. The design of motivational agents and avatars. Educational Technology Research and Development 59, 2 (2011), 291-300.

[5] Konstantinos Bousmalis, Marc Mehu, and Maja Pantic. 2009. Spotting agreement and disagreement : A survey of nonverbal audiovisual cues and tools. In 2009 $3 r d$ international conference on affective computing and intelligent interaction and workshops. IEEE, 1-9.

[6] Joao Paulo Cabral, Benjamin R Cowan, Katja Zibrek, and Rachel McDonnell. 2017. The Influence of Synthetic Voice on the Evaluation of a Virtual Character.. In INTERSPEECH. 229-233.

[7] Scotty D Craig and Noah L Schroeder. 2017. Reconsidering the voice effect when learning from a virtual human. Computers \& Education 114 (2017), 193-205.

[8] Joana Galvão Gomes da Silva, David J Kavanagh, Tony Belpaeme, Lloyd Taylor, Konna Beeson, and Jackie Andrade. 2018. Experiences of a motivational interview delivered by a robot : qualitative study. Fournal of medical Internet research 20, 5 (2018), e116.

[9] Cally A Davies, John C Spence, Corneel Vandelanotte, Cristina M Caperchione, and W Kerry Mummery. 2012. Meta-analysis of internet-delivered interventions to increase physical activity levels. International fournal of Behavioral Nutrition and Physical Activity 9, 1 (2012), 1-13.

[10] J Larry Durstine, Benjamin Gordon, Zhengzhen Wang, and Xijuan Luo. 2013 Chronic disease and the link to physical activity. Journal of Sport and Health Science 2, 1 (2013), 3-11.

[11] Ellen C Garbarino and Julie A Edell. 1997. Cognitive effort, affect, and choice. fournal of consumer research 24, 2 (1997), 147-158.

[12] Areti Goulati and Dalila Szostak. 2011. User experience in speech recognition of navigation devices : an assessment. In Proceedings of the 13th International Conference on Human Computer Interaction with Mobile Devices and Services. 517-520.

[13] Marcel Heerink, Ben Krose, Vanessa Evers, and Bob Wielinga. 2009. Measuring acceptance of an assistive social robot : a suggested toolkit. In RO-MAN 2009-The 18th IEEE International Symposium on Robot and Human Interactive Communication. IEEE, 528-533.

[14] Toshikazu Kanaoka and Bilge Mutlu. 2015. Designing a motivational agent for behavior change in physical activity. In Proceedings of the 33rd Annual ACM Conference Extended Abstracts on Human Factors in Computing Systems. 14451450 .

[15] JF Lemoine and E Cherif. 2015. Quel est le meilleur agent virtuel pour mon site? Une étude exploratoire des différentes caractéristiques anthropomorphiques. XXXème Congrès International de l'Association Française du Marketing (AFM) 15 (2015).

[16] Christine Lisetti, Reza Amini, Ugan Yasavur, and Naphtali Rishe. 2013. I can help you change! an empathic virtual agent delivers behavior change health interventions. ACM Transactions on Management Information Systems (TMIS) 4 4 (2013), 1-28.

[17] Rosemarijn Looije, Mark A Neerincx, and Fokie Cnossen. 2010. Persuasive robotic assistant for health self-management of older adults : Design and evaluation of social behaviors. International fournal of Human-Computer Studies 68, 6 (2010), 386-397.

[18] Brad Lundahl and Brian L Burke. 2009. The effectiveness and applicability of motivational interviewing : A practice-friendly review of four meta-analyses. Fournal of clinical psychology 65, 11 (2009), 1232-1245.

[19] WR Miller, J C'de Baca, DB Matthews, and PL Wilbourne. 2001. Personal values card sort. Albuquerque, NM : University of New Mexico (2001).

[20] William R Miller and Stephen Rollnick. 2012. Motivational interviewing : Helping people change. Guilford press.

[21] Theresa B Moyers, Lauren N Rowell, Jennifer K Manuel, Denise Ernst, and Jon M Houck. 2016. The motivational interviewing treatment integrity code (MITI 4) rationale, preliminary reliability and validity. Fournal of substance abuse treatment 65 (2016), 36-42.

[22] Mohsen Naghavi, Amanuel Alemu Abajobir, Cristiana Abbafati, Kaja M Abbas, Foad Abd-Allah, Semaw Ferede Abera, Victor Aboyans, Olatunji Adetokunboh, Ashkan Afshin, Anurag Agrawal, et al. 2017. Global, regional, and national agesex specific mortality for 264 causes of death, 1980-2016: a systematic analysis for the Global Burden of Disease Study 2016. The Lancet 390, 10100 (2017), $1151-1210$.

[23] Kristine L Nowak, Mark A Hamilton, and Chelsea C Hammond. 2009. The effect of image features on judgments of homophily, credibility, and intention to use as avatars in future interactions. Media Psychology 12, 1 (2009), 50-76.
[24] Stefan Olafsson, Teresa O'Leary, and Timothy Bickmore. 2019. Coerced Changetalk with Conversational Agents Promotes Confidence in Behavior Change. In Proceedings of the 13th EAI International Conference on Pervasive Computing Technologies for Healthcare. 31-40.

[25] Stefan Olafsson, Byron Wallace, and Timothy W Bickmore. 2020. Towards a Computational Framework for Automating Substance Use Counseling with Virtual Agents.. In AAMAS. 966-974.

[26] Rita Orji and Karyn Moffatt. 2018. Persuasive technology for health and wellness : State-of-the-art and emerging trends. Health informatics journal 24, 1 (2018), 66-91.

[27] SoHyun Park, Jeewon Choi, Sungwoo Lee, Changhoon Oh, Changdai Kim, Soohyun La, Joonhwan Lee, and Bongwon Suh. 2019. Designing a chatbot for a brief motivational interview on stress management : qualitative case study. fournal of medical Internet research 21, 4 (2019), e12231.

[28] Simon Provoost, Ho Ming Lau, Jeroen Ruwaard, and Heleen Riper. 2017. Embodied conversational agents in clinical psychology : a scoping review. fournal of medical Internet research 19, 5 (2017), e151.

[29] Chao Qu, Willem-Paul Brinkman, Yun Ling, Pascal Wiggers, and Ingrid Heynderickx. 2014. Conversations with a virtual human : Synthetic emotions and human responses. Computers in Human Behavior 34 (2014), 58-68.

[30] J. Ricci and L. Gagnon. 2016. Examen périodique de santé. Questionnaire Ricci Gagnon. Test d'auto-évaluation de l'activité physique. Retrieved from https ://www.ameli.fr/sites/default/files/questionnaire-activite-physique cpam haute - savoie.pdf (2016).

[31] Daniel Schulman, Timothy W Bickmore, and Candace L Sidner. 2011. An Intelligent Conversational Agent for Promoting Long-Term Health Behavior Change Using Motivational Interviewing.. In AAAI Spring Symposium : AI and Health Communication.

[32] Rebecca M Shingleton and Tibor P Palfai. 2016. Technology-delivered adaptations of motivational interviewing for health-related behaviors : A systematic review of the current research. Patient education and counseling 99, 1 (2016), 17-35.

[33] Nick Yee, Jeremy N Bailenson, and Kathryn Rickertsen. 2007. A meta-analysis of the impact of the inclusion and realism of human-like faces on user experiences in interfaces. In Proceedings of the SIGCHI conference on Human factors in computing systems. 1-10. 